\title{
Vantagens da implantação do prontuário eletrônico na segurança do paciente e na otimização do trabalho do farmacêutico hospitalar.
}

\author{
Advantages of implanting the electronic medical record in patient \\ safety and optimizing the work of the hospital pharmacist.
}

Recebido em: 07/02/2017

Aceito em: 29/05/2017

Valdinara CRIPPA ${ }^{1}$; Diego Roberto Colombo DIAS ${ }^{2}$

${ }^{1}$ Universidade Federal de São Paulo.Rua Sena Madureira, 1500, Vila Clementino.

São Paulo, SP, Brasi. ${ }^{2}$ Universidade Federal de São João Del Rei.

Praça Frei Orlando, 170, Centro. São João Del Rei, MG, Brasil.

E-mail:valdinara_crippa@hotmail.com

\begin{abstract}
In order to analyse the contributions of the electronic medical record to the patient safety during the use of drugs in hospitals and to the optimization of the work of Pharmacist, a systematic review of original articles was presented, showing the benefits of electronic medical records in hospitals to prescription safety. Relevant studies were identified through the PUBMED, LILACS, MEDLINE and SciELO databases. A total of 99 articles were returned, and 10 of them were selected for discussion, according the established criteria. The analysis pointed to the use of electronic medical records in hospitals as favorable to improve the support for clinical decision and medication monitoring, reduction of errors, timely interventions and organization of work processes. At the end, the conclusion wast the electronic medical record contributes to the quality and efficiency of patients care, and optimizes the work of Pharmacists and other Health professionals in the hospital network.
\end{abstract}

Keywords: Electronic medical records; pharmaceutical; hospital; patient safety; informatization; information technology.

\section{RESUMO}

Com o objetivo de analisar as contribuições do prontuário eletrônico na garantia da segurança do paciente no uso de medicamentos em hospitais, e seu reflexo na otimização do trabalho do farmacêutico hospitalar, foi feita uma revisão sistemática de artigos originais apresentando benefícios referentes à segurança da prescrição e administração de medicamentos com o uso do prontuário eletrônico em hospitais. Estudos relevantes foram identificados por meio das bases de dados PUBMED, LILACS, MEDLINE e Scielo. Foram retornados 99 artigos, dos quais 10 que melhor atenderam aos critérios estabelecidos, foram selecionados para discussão. A análise apontou o uso do prontuário eletrônico em hospitais como favorável para viabilizar maior apoio à decisão clínica e monitoramento de medicamentos, redução de erros, realização de intervenções atempada e maior agilidade e organização dos processos de trabalho, concluindo que o prontuário eletrônico contribui para a qualidade e eficiência da assistência prestada e otimiza o trabalho dos farmacêuticos e demais profissionais da rede hospitalar.

Palavras-chave: Prontuário eletrônico; farmacêutico; hospital; segurança do paciente; informatização; tecnologia da informação. 


\section{INTRODUÇÃO}

O prontuário do paciente é um documento obrigatório, de caráter legal e sigiloso, fundamental para a prestação da assistência ao paciente, construído colaborativamente pela equipe multiprofissional de saúde. Contém informações sobre a história clínica e social do paciente e a própria assistência a ele prestada, permitindo a comunicação entre os membros da equipe e acompanhamento do paciente (1). Há necessidade de garantir a qualidade e a segurança dessa informação, bem como melhorar o fluxo da assistência prestada nas rotinas hospitalares, e foi com esse intuito que foi desenvolvido o Prontuário Eletrônico do Paciente (PEP) em substituição ao prontuário em papel. O PEP é uma ferramenta informatizada que possibilita a integração e interação facilitada e segura dos dados relacionados ao paciente e das ações produzidas na assistência ao mesmo $(2,3)$.

Com o PEP é possível eliminar algumas das dificuldades e dos riscos aos quais prontuário em papel está exposto, tais como extravio, quebra de privacidade, degradação, dificuldade de recuperação e compartilhamento de informações entre profissionais, repetição de coleta de dados, dificuldades de armazenamento, entre outros. Várias outras vantagens são possíveis com a utilização do PEP, tais como: acesso rápido e simultâneo às informações por diversos profissionais de saúde, legibilidade absoluta das informações, organização sistemática das informações e possibilidade de emissão de relatórios, integração com outros sistemas de informação, maior agilidade na solicitação e verificação de exames e medicações, acesso a bases de apoio à decisão clínica, sistema de redução de erros, entre outros. A qualidade da informação é melhorada com a implantação do prontuário eletrônico, consequentemente, a assistência e a segurança na prestação dos cuidados ao paciente também tornam-se melhores (3). A implantação do PEP nas unidades hospitalares permite, além da melhora na qualidade do atendimento prestado ao paciente, uma mudança nos processos de trabalho, aumentando a produtividade e otimizando o trabalho dos profissionais de saúde. Além disso, melhora a integração entre áreas assistenciais e de gestão hospitalar.

No âmbito de atuação de farmacêuticos hospitalares o PEP fornece ferramentas que os auxiliam na execução de suas atividades, contribuindo para o cumprimento dos requisitos do Protocolo de Segurança do Paciente coordenado pelo Ministério da Saúde e Agência Nacional de Vigilância Sanitária (ANVISA), nos critérios de segurança na prescrição, dispensação, uso e administração de medicamentos, uma vez que as falhas no processo de utilização de medicamentos são consideradas importantes fatores contribuintes para os eventos adversos significativos (4).

A incorporação de meios para reduzir erros com medicações, como uso de recursos de tecnologia da informação, facilidade de acesso a informações sobre os medicamentos, desenvolvimento de protocolos internos de padronização de medicamentos e de diluições, educação permanente e acompanhamento das práticas profissionais em todas as etapas que envolvem o medicamento, reduz a probabilidade de falhas e aumenta a chance de interceptá-las antes de resultar em prejuízo ao paciente, sendo este um dos princípios do protocolo de segurança do paciente e uma das atividades primordiais do farmacêutico nas unidades hospitalares (4).

O farmacêutico hospitalar, ainda, em sua prática clínica, atua integrado à equipe multiprofissional melhorando a utilização da farmacoterapia e aprimorando os conceitos de segurança no uso de medicamentos a partir da identificação e resolução de problemas relacionados à terapia medicamentosa, diminuindo a incidência de erros de medicação, reações adversas, interações e incompatibilidades medicamentosas, aumentando a qualidade da atenção ao paciente $(4,5)$.

Neste contexto, ferramentas integradas a sistemas de prontuários eletrônicos com suporte de apoio à decisão clínica com alertas e informações sobre indicações, dose, posologia, contraindicações, interações e incompatibilidades medicamentosas, apoiando a correta prescrição dos medicamentos facilita o trabalho do farmacêutico. Ainda, ferramentas que permitem maior eficiência nas atividades de reconciliação medicamentosa, orientações em alta hospitalar, triagens de farmacovigilância e acompanhamento farmacoterapêutico de medicamentos potencialmente perigosos prescritos aumentam a segurança do paciente.

Além disso, o PEP viabiliza a disponibilidade e a organização das informações dos pacientes, necessárias para o acompanhamento farmacoterapêutico e realização das intervenções $(1,6)$.

Contudo, sua implementação nas unidades hospitalares querer mudanças dos processos de trabalho, abrindo uma discussão histórica das rotinas médico-assistenciais, conceitos éticos, informatização de setores e treinamento de todos os envolvidos no processo (2).

Considerando essas vantagens da implementação do PEP, o presente estudo tem como objetivo analisar as contribuições do prontuário eletrônico para a garantia 
de segurança do paciente no uso de medicamentos em hospitais, e seu reflexo na otimização do trabalho do farmacêutico hospitalar.

\section{MÉTODO}

O presente estudo consiste em uma revisão sistemática, na qual para obtenção dos artigos a serem analisados foram utilizados os descritores: PRONTUÁRIO ELETRÔNICO, TECNOLOGIA DA INFORMAÇÃO, INFORMATIZAÇÃO, HOSPITAL, FARMACÊUTICO, SEGURANÇA DO PACIENTE, nas línguas portuguesa, inglesa e espanhola, realizando a busca nas bases de dados PUBMED, LILACS, MEDLINE e Scielo. O período de pesquisa compreendeu os anos de 2000 até 2016, visando encontrar o maior número de artigos que relacionasse o uso do prontuário eletrônico com a atividade do farmacêutico hospitalar. Referências bibliográficas de artigos e outras revisões também foram pesquisadas a fim de complementar a conceituação de termos e agregar informações importantes para o desenvolvimento do trabalho.

Os critérios de inclusão dos estudos selecionados foram: artigos originais e completos nas línguas portuguesa, inglesa e espanhola, com os resumos disponíveis nas bases de dados selecionadas, que apresentassem experiências de profissionais de saúde no uso de prontuário eletrônico e que abordassem as vantagens da utilização do prontuário eletrônico para segurança da prescrição, uso e administração de medicamentos em hospitais. Foram excluídos os estudos que não incluíssem o profissional farmacêutico nas pesquisas ou que não mencionassem atividades inerentes ao campo de atuação desse profissional, também excluídos os artigos curtos e de revisão de literatura.

A partir da string de busca foram retornados 99 artigos, dos quais foram selecionados para o desenvolvimento do trabalho os 10 artigos que melhor atenderam aos critérios de inclusão e mais se adequaram ao objetivo da presente revisão. Para análise e seleção dos artigos que melhor atenderam os critérios de inclusão foi utilizada uma tabela com todos os artigos retornados na busca contendo título do artigo, resumo e fonte consultada.

\section{RESULTADOS E DISCUSSÃO}

Todos os artigos selecionados apresentaram o uso de sistemas eletrônicos como necessário para a detecção mais eficaz de erros de medicação. O Quadro 1 mostra os 10 artigos selecionados no estudo, sendo estes os que melhor relacionaram a implantação do PEP com a segurança do paciente, aliado às atividades do profissional farmacêutico em unidades hospitalares. Entre os dados relevantes pode ser destacada a análise de Westbrook e cols. (2015), que identificaram 12.567 erros de prescrição hospitalar, das quais 539 eram clinicamente importantes, mas apenas 13 foram detectados pela equipe de saúde, afirmando que o uso de abordagens eletrônicas poderia ter detectado os 421 erros de prescrição não detectados pela equipe (7). Pode ser observado que erros de prescrição com o potencial de causar danos muitas vezes passam despercebidos pela observação humana, e por isso a implantação de sistemas eletrônicos de informações clínicas podem apoiar de forma mais eficaz a detecção de erros de medicação. Como mostraram Shah e cols. (2006), a geração de alertas em prescrições informatizadas dentro de um PEP projetam apoio à decisão médica com altos índices de aceitação por parte da equipe médica. No período de 6 meses, 5.182 prescrições de medicamentos com alertas críticos de alta gravidade foram interrompidas, e os alertas tiveram $67 \%$ de aceitação por parte da equipe médica (10).

Outro dado importante foi o apontado no estudo de Galanter e cols. (2005): após a implantação do PEP e a aplicação de alertas em prescrições, a probabilidade de um paciente com insuficiência renal receber uma dose contraindicada de um fármaco diminuiu de $89 \%$ para $47 \%$ em seu estudo, demonstrando que os alertas nos sistemas de prontuário eletrônicos são eficazes na diminuição de prescrição e administração de medicamentos contraindicados (16).

Em erros de reconciliação medicamentosa, o estudo de Schnipper e cols. (2009) mostrou que as divergências de medicações com potencial para provocar dano ao paciente diminuiram de 1,44/paciente (grupo controle) para 1,05/paciente (grupo intervenção) com o uso de ferramenta informatizada (9). Ainda nesse sentido, o estudo de Poon e cols. (2006) mostrou que o desenvolvimento de aplicações informatizadas para a reconciliação medicamentosa além de prevenir erros com medicamentos na admissão e alta hospitalar de pacientes, permitiu a coleta de dados e transcrição dos cuidados, facilitando também a comunicação interdisciplinar entre médicos, enfermeiros e farmacêuticos (12), visto que, após a utilização do Sistema, a percepção de melhorias de processos e a aceitação por parte dos profissionais são significativas. 
Esses relatos de experiências exitosas após a implantação de prontuários e sistemas eletrônicos de registros também foram observados no estudo de Câneo \& Rondina (2014), no qual profissionais de instituições hospitalares brasileiras mencionaram, como vantagens desses processos informatizados, a redução no tempo de atendimento, o acesso fácil e rápido ao histórico do paciente, com organização clara das informações, melhorando o planejamento terapêutico e tomada de decisão; o compartilhamento simultâneo das informações com toda equipe multidisciplinar, a prevenção de erros de diagnóstico e prescrição medicamentosa, melhorando a qualidade dos processos e garantindo maior efetividade dos cuidados (14), como também observado por Farias e cols. (2011) em relatos de profissionais de saúde de instituições hospitalares no Brasil e na Espanha, cuja percepção da implantação dessa tecnologia foi de contribuição para todos os processos de gestão e assistência hospitalar (15), apontando também o fato de que, quanto maior o domínio do profissional com a informática melhor foi o nível de aceitação e percepção da contribuição do prontuário eletrônico para o desempenho das atividades.

Quadro 1. Artigos selecionados que apresentam dados que evidenciam resultados positivos com o uso de prontuário eletrônico na garantia da segurança da prescrição e uso adequado de medicamentos em unidades hospitalares.

\begin{tabular}{|c|c|}
\hline Título e Ano & Objetivo \\
\hline $\begin{array}{l}\text { What are incident } \\
\text { reports telling us? A } \\
\text { comparative study at } \\
\text { two Australian hospitals } \\
\text { of medication errors } \\
\text { identified at audit, } \\
\text { detected by staff and } \\
\text { reported to an incident } \\
\text { system. } 2015 \text { (7) }\end{array}$ & $\begin{array}{l}\text { Comparar os erros de medicação } \\
\text { identificados na auditoria e de } \\
\text { observação com relatórios medicação } \\
\text { incidentes; identificar as diferenças } \\
\text { entre dois hospitais na frequência } \\
\text { relatório sobre o incidente e as taxas } \\
\text { de erros de medicação; identificar } \\
\text { prescrição taxas de detecção de erro } \\
\text { pela equipe. }\end{array}$ \\
\hline
\end{tabular}

Electronic surveillance and pharmacist intervention for vulnerable older inpatients on high-risk medication regimens. 2014 (8)
Desenvolver e avaliar uma ferramenta eletrônica para auxiliar os farmacêuticos clínicos com a revisão de medicamentos potencialmente perigosos (PIM) em adultos idosos hospitalizados.
Effect of an electronic medication reconciliation application and process redesign on potential adverse drug events: a cluster-randomized trial. 2009 (9)
Resultados

Fonte

Um total de 12.567 erros de prescrição foram identificados no estudo, dos quais 539 erros de prescrição clinicamente importantes, e 13 foram relatados pela equipe institucional. Um total de 2.043 administrações de medicamentos continham $\geq 1$ erros, porém nenhum tinha um relatório de incidente. Novas abordagens, incluindo mineração de dados eletrônicos e informações clínicas nos sistemas são necessárias para apoiar a detecção mais eficaz de erros de medicação.

Participaram do estudo adultos hospitalizados com 65 anos ou mais, internados em um hospital universitário, durante um período de 3 semanas, aos quais tinham sido administrados pelo menos um medicamento da lista de 240 medicamentos potencialmente perigosos (PIM). Um painel informatizado sinalizava os indivíduos com prescrição de anticolinérgicos em alta dosagem e em administração de narcóticos e benzodiazepínicos cumulativo de 48 horas. 0 painel sinalizou 179 de 797 indivíduos (22\%) e 485 pares de medicação-participante para avaliação por parte do farmacêutico clínico. Vinte e dois participantes que receberam 40 pedidos de medicações inadequadas foram avaliados quanto à necessidade de intervenção. 0s médicos ratificaram 31 das 40 recomendações farmacêuticas (78\%).

Medir o impacto de uma tecnologia da informação na intervenção em reconciliação medicamentosa, baseada em divergências de medicação com potencial para provocar danos [potenciais eventos adversos (PAdES)].
Foram incluídos no estudo 322 pacientes admitidos por 14 equipes médicas, dos quais um histórico de medicação pudesse ser obtido antes da alta. Para o grupo intervenção foi realizada a reconciliação medicamentosa por meio de ferramenta informatizada, e os processos envolveram médicos, enfermeiros e farmacêuticos. Entre 160 pacientes do grupo controle, houve 230 potenciais efeitos adversos (PAdES) (1,44 por paciente), enquanto que entre 162 pacientes de intervenções havia 170 PAdES (1,05 por paciente), associando a ferramenta com uma diminuição nas discrepâncias de medicações não intencionais com potencial para dano ao paciente. 
Improving acceptance of computerized prescribing alerts in ambulatory care. 2006 (10)
Melhorar a aceitação clínica dos alertas de medicamentos em prescrições eletrônicas, por meio da concepção de alertas seletivos para a configuração de atendimento ambulatorial, designando apenas alertas críticos de alta gravidade minimizando assim as interrupções do fluxo de trabalho.

Avaliar os benefícios de sistema médico computadorizado (POE) e o registro eletrônico de administração de medicamentos (EMAR) na entrega de cuidados em saúde em unidades de internamento de um sistema acadêmico de saúde.
Os alertas foram apresentados aos médicos utilizando a prescrição dentro de um registro médico eletrônico em 31 áreas de atendimento ambulatorial de Boston. Foram detectados 18.115 alertas de medicamentos com inconsistências de prescrição, gerados durante o período de estudo (seis meses). Destes, 12.933 (71\%) não foram interrompidos e $5.182(29 \%)$ interrompidos. Dos alertas interrompidos, $67 \%$ foram aceitos. As razões para substituições variaram para cada fármaco e categoria de alerta.

Reduções estatisticamente significativas foram observadas após a implementação do POE para tempos de turnaround de medicação $(64 \%$, de 05h:28min. para 01h:51min.), tempos de conclusão de procedimento de radiologia $(43 \%$, de $07 \mathrm{~h}: 37 \mathrm{~min}$. para $04 \mathrm{~h}: 21 \mathrm{~min}$.), e resultado de laboratório (25\%, 31min:03seg. para 23min:04seg.). Além disso, POE combinado com EMAR eliminou todos os erros médicos e transcrição de enfermagem. Foram observadas $43 \%$ e $26 \%$ de melhora na autenticação final por médicos no sistema The Ohio State University Hospitals (OSUH) e James Cancer Hospital (James), respectivamente. 0 parâmetro gravidadeestadia diminuiu em OSUH (pré-POE, 3,91 dias; pós-P0E, 3,71 dias), mas não de forma significativa em James (pré-POE, 3,68 dias; pós-P0E, 3,61 dias). 0 custo total por internação diminuiu nos serviços selecionados, mas não se alterou significativamente entre uma destas instituições (OSUH: pré-POE, 5.697 dólares; pós-POE, 5.661 dólares; James: pré-POE, 6.427 dólares; pós-POE, 6.518 dólares).

Dos 216 pacientes internados nas equipes-piloto do estudo, 166 (78\%) tinham uma lista de medicamentos de pré-admissão (PAML) criada a partir de um aplicativo informatizado. No geral, os profissionais que participaram das sessões de feedback dos testes-piloto, 41 médicos, 310 enfermeiros e 75 farmacêuticos que participaram dos pilotos, revelaram que a maioria dos usuários considerou a interface intuitiva e muitos foram capazes de usar o sistema sem qualquer formação, relatando que o novo sistema ajudou a evitar vários erros de medicação. Alguns médicos relataram que foi tão útil que eles se sentiram desconfortáveis quando se deslocaram para setores sem o PAML disponível, uma vez que a ferramenta otimizou o processo de reconciliação medicamentosa.

A proporção de pacientes com insuficiência cardíaca de todas as causas de reinternações com 30 dias foi, respectivamente, $30 \%, 29 \%$ e $32 \%$ para aqueles com alta do hospital com completa abrangência de sistemas de registro eletrônico do paciente, com sistemas parciais, e com sistemas não abrangentes, não sendo significativamente importantes. $\mathrm{Na}$ análise de sobrevida multivariada final, os beneficiários que receberam alta de hospitais relatando plena implementação de registros médicos eletrônicos abrangentes contra nenhuma implementação tinham a mesma probabilidade de ser readmitido dentro de 30 dias por qualquer causa.
PUBMED

\section{PUBMED}

PUBMED electronic medical record implementation levels not associated with 30-day all-cause readmissions within Medicare beneficiaries (13)
Comparar taxas de incidência de de pacientes com insuficiência cardíaca que receberam alta dos hospitais com plena implementação de sistemas de registro eletrônico do paciente com aqueles sem a ferramenta. 
Prontuário Eletrônico do Paciente: conhecendo as experiências de sua implantação. 2014 (14)
Avaliar as principais vantagens e desvantagens da adoção de um sistema de prontuários eletrônicos, tanto para a equipe médica quanto para o paciente.
Adoção de prontuário eletrônico do paciente em hospitais universitários de Brasil e Espanha. A percepção de profissionais de saúde. 2011 (15)
Descrever a percepção de profissionais de saúde quanto ao prontuário eletrônico do paciente na gestão de hospitais e o impacto dessa tecnologia no trabalho em hospitais universitários do Brasil e Espanha.
Foram selecionados e analisados relatos de

LILACS experiências de implantação de prontuários eletrônicos em 12 instituições de saúde brasileiras. Em 9 foram observadas redução no tempo de atendimento e facilidade na consulta de dados em atendimentos futuros. Sete apontaram o acesso rápido ao histórico do paciente, processamento contínuo e atualizado dos dados, melhora do planejamento, atendimento e controle hospitalar; 6 mencionaram os benefícios de compartilhamento das informações por diversos profissionais, organização sistemática, objetiva e clara dos dados, auxílio no processo de tomada de decisão e na efetividade do cuidado, e o uso das informações para estudos sóciodemográficos e epidemiológicos e de avaliação da qualidade. Cinco destacaram a integração com outros sistemas de informação, prevenção de erros de diagnóstico, prescrição e interação de medicamentos e a eliminação de duplicidade de dados e pedidos de exames. A única desvantagem citada por mais de $50 \%$ dos relatos foi a resistência dos profissionais de saúde quanto ao uso de novas tecnologias, devida à falta de domínio de informática.

Os resultados mostraram que: esta tecnologia é percebida como um mecanismo que contribui com a gestão e a assistência hospitalar mediante mecanismos de controle; os respondentes com maior domínio de informática concordaram mais do que aqueles com menor domínio a respeito do impacto desse prontuário no respectivo desempenho; e houve diferenças nas médias de percepção do papel desse prontuário no desempenho quando considerado o tempo de serviço, sendo o maior nível de concordância com o PEP quanto menor o tempo de serviço. Estudos adicionais são sugeridos ao final.

A probabilidade de um paciente que recebe pelo menos uma dose de fármaco contraindicado, por meio de prescrição médica, diminuiu de $89 \%$ para $47 \%$ após aplicação de alertas. A análise dos alertas vistos por médicos mostrou que 0 cumprimento de alerta foi maior no sexo masculino ( $57 \%$ vs. $38 \%$ ), aumentou com a duração da formação dos médicos, e aumentou também quando tratavam-se de alertas para prescrição de pacientes com agravamento da função renal.
Scielo e LILACS
A trial of automated decision support alerts for contraindicated computerized physician order entry. 2005 (16)
Avaliar a utilidade de alertas uso de fármacos contraindicados em paciente com insuficiência renal, bem como analisar os fatores que podem desempenhar um papel no descumprimento de recomendações de alerta entre a equipe médica.
O auxílio da ferramenta eletrônica também é importante para auxiliar farmacêuticos clínicos na identificação e revisão de medicamentos potencialmente perigosos em pacientes hospitalizados, como mostrado no estudo de Peterson e cols. (2014), que mostrou 179 sinalizações precisamente detectadas por um painel no sistema informatizado e, destas, 40 necessitaram de intervenções farmacêuticas, das quais 31 foram aceitas e ratificadas pelos médicos (8). Em relação às taxas de readmissão, a implantação do prontuário eletrônico não mostrou resultados significativos na redução. em comparação com hospitais que não possuíam prontuário eletrônico, e a falta do apoio de sistemas informatizados à decisão clínica por meio pode ser associada, mas não é fonte causal para as readmissões. O estudo não pode considerar as outras fontes de heterogeneidade para melhores evidências desse fator influenciador. 
Mas em relação à melhoria do fluxo de trabalho, a implantação do prontuário eletrônico apresentou resultados favoráveis, como observado no estudo de Mekhjian e cols. (2011), onde a otimização de tempo de turnarou$n d$ de medicação, por exemplo, foi um benefício adquirido com a implementação do PEP de forma completa, passando de 05h:28min. para 01h:51min (11).

A qualidade na informação, proporcionada pelo prontuário eletrônico e suas ferramentas de monitoramento de medicamentos e o apoio à decisão clínica, viabilizam ao profissional de saúde melhores condições de atuação baseada em princípios científicos, melhor planejamento e avaliação da tomada de decisão, garantindo uma maior qualidade do cuidado prestado (17). O PEP fornece também estrutura para melhoras na segurança do paciente e na pontualidade do atendimento (11), favorecendo mudanças culturais e no fluxo de trabalho das instituições hospitalares. Além disso, as reduções de erros de transcrição e comunicação dos resultados, e intervenções a tempo suportam a visão que o PEP proporciona um bom retorno sobre o investimento, mesmo que o tempo e os custos com a internação não diminuam significativamente $(11,13)$.

Melhorar a eficiência dos cuidados e garantir o tratamento adequado é primordial em serviços de saúde, enfatizando o fato de que a farmacoterapia é um dos principais tipos de tratamentos utilizados em unidades hospitalares, ressaltando deste modo, a importância da adoção de sistemas que garantam o seu uso racional e a redução de erros com medicamentos (18). Minimizar os riscos para erros relacionados à administração de medicamentos é garantir maior segurança ao paciente hospitalizado, sendo uma das metas internacionais de padrões de qualidade e segurança do paciente (4). Os erros de medicação podem causar danos à saúde, podendo afetar o tratamento do paciente, provocar reações adversas significativas, prolongar o período de internação, ou até mesmo, em alguns casos serem letais, e ainda gerar relevantes repercussões econômicas e sociais, que interferem diretamente a vida dos pacientes, dos profissionais de saúde e da instituição.

Neste sentido, a tecnologia da informação tem sido identificada como um componente importante de melhoria. Estudos tem demonstrado que a implantação de prontuário eletrônico do paciente permite diminuir os erros, melhorar o processo organizacional, diminuir custos e apoiar a tomada de decisões e a execução dos cuidados de forma mais segura (19).

Falar em segurança do paciente relacionada a medicamentos remete à redução dos riscos de erros em todas as etapas do fluxo da terapia medicamentosa, principalmente nas etapas de prescrição, dispensação e administração, como no estudo realizado por Allard e cols. (2002que observaram altos índices de erros em prescrição (49\%), dispensação (14\%) e na administração $(26 \%)$ ) (20). Diante disso, foi constatado, na literatura, que o sistema de prontuário eletrônico do paciente é um modelo que pode contribuir fortemente para a redução desses erros com medicações em até $50 \%(21)$.

Uma pesquisa realizada por Devine e cols. (2010), que compararam prescrições manuais e eletrônicas, evidenciou que a frequência de erros após implantação de prontuário com sistema de prescrição eletrônica diminuiu de $18,2 \%$ para $8,2 \%$. As maiores reduções foram observadas em probabilidades de ajustes de erros por ilegibilidade (97\%), uso de abreviaturas inadequadas (94\%) e falta de informação (85\%) (22).

Com o prontuário eletrônico ainda é possível garantir o suporte à decisão clínica no que diz respeito a medicamentos, alterações laboratoriais, diretrizes, alertas de interação medicamentosa importante, de alergias, opções de frequência e dose máxima diária dos medicamentos, sugerindo alternativas e melhores estruturações da prescrição médica, a fim de gerar evitar possíveis erros (23).

Foi verificado que informações clínicas e alertas nos sistemas de prescrição informatizada em prontuários eletrônicos apoiam de forma eficaz a detecção de erros de medicação, podendo realizar a intervenção antes de provocar o dano. Também é possível a realização, de forma mais otimizada, da vigilância dos erros, do monitoramento das prescrições de medicamentos potencialmente perigosos e condições clínicas que necessitam de maior acompanhamento farmacoterapêutico realizado pelos profissionais farmacêuticos.

Os benefícios proporcionados pela implantação do prontuário eletrônico, observados nos estudos analisados, apontaram para fato de essa ser uma tecnologia utilizada para facilitar e garantir a prescrição, dispensação e administração mais segura dos medicamentos e melhorar a comunicação e o fluxo de trabalho para todas as equipes envolvidas.

As contribuições dos resultados da pesquisa são aplicáveis ao aprimoramento dos estudos e práticas de implantação de prontuários eletrônicos do paciente em unidades hospitalares e no campo de atuação do farmacêutico, incentivando à segurança do paciente e os investimentos nessa área, a fim de minimizar 
os erros com medicamentos e contribuir com a otimização da terapia medicamentosa e dos processos de trabalho.

\section{CONCLUSÃO}

Por meio desta pesquisa foi possível concluir que o PEP é uma ferramenta que contribui para a qualidade da assistência prestada em hospitais, tornando mais eficiente os processos de trabalho e atuando como media-

\section{REFERÊNCIAS}

1. Galvão MCB, Ricarte ILM. O prontuário eletrônico do paciente no século XXI: contribuições necessárias da ciência da informação. R. Ci. Inf. E Doc. 2011;2(2): 77100. DOI: 10.11606/issn.2178-2075.v2i2p77-100

2. Bezerra SM. Prontuário Eletrônico do Paciente: uma ferramenta para aprimorar a qualidade dos serviços de saúde. Meta: Avaliação. 2009; 1(1):73-82. DOI: 10.22347/2175-2753v1i1.12

3. Patrício CM, Maia MM, Machiavelli JL, Navaes MA. O prontuário eletrônico do paciente no sistema de saúde brasileiro: uma realidade para os médicos? Sci. Med. 2011; 21(3):121-131.

4. BRASIL. Ministério da Saúde. Programa Nacional de Segurança do Paciente. Protocolo de segurança na prescrição, uso e administração de medicamentos. Brasília: Ministério da Saúde, 2013. Disponível em: http:// proqualis.net/sites/proqualis.net/files/000002490IQmw D8.pdf

5. Reis AMM. Atenção Farmacêutica e promoção do uso racional de medicamentos. Centro de Estudos em Atenção Farmacêutica. 2015. Disponível em: http://www. ceatenf.ufc.br/Artigos/ATENFAR\%20e\%20URM\%20 Adriano\%20Max.pdf

6. Pinto VB. Prontuário eletrônico do paciente: documento técnico de informação e comunicação do domínio da saúde. R. Eletr. Bibliotecon. Cien. Inform. 2006; 21(1):3448. DOI: $10.5007 / 1518-2924.2006 v 11 n 21 \mathrm{p} 34$.

7. Westbrook JI, Li L, Lehnbom EC, Baysari MT, Braithwaite J, Burke R, Conn C, Day R. What are incident reports telling us? A comparative study at two Australian hospitals of medication errors identified at audit, detected by staff and reported to an incident system. Int. J. Qual. Health Care. 2015; 27(1):1-9. DOI: 10.1093/intqhe/ mzu098.

8. Peterson JF, Kripalani S, Danciu I, Harrell Dd, Marvanova M, Mixon AS, Rodriguez C, Powers JS. Electronic surveillance and pharmacist intervention for vulnerable older inpatients on high-risk medication regimens. J. Am. dor na redução de erros de medicamentos, melhorando as decisões clínicas, transformando comportamentos e otimizando o trabalho dos farmacêuticos e dos demais profissionais da rede hospitalar.

Foi possível notar a contribuição do prontuário eletrônico na agilidade da assistência hospitalar, na organização das rotinas de trabalho, no gerenciamento dos serviços e registro seguro das ações dos profissionais de saúde em hospitais, mostrando que a implantação desse sistema, após período de adaptação, traz mais benefícios que prejuízos à equipe de saúde e aos pacientes.

Geriatr. Soc. 2014. 62(11):2148-2152. DOI: $10.1111 /$ jgs. 13057.

9. Schnipper JL, Hamann C, Ndumele CD, Liang CL, Carty MG, Karson AS, Bham I, Coley CM, Poon E, Turchin A, Labonville SA, Diedrichsen EK, Lipsitz S, Broverman CA, McCarthy P, Gandhi TK. Effect of an electronic medication reconciliation application and process redesign on potential adverse drug events: a cluster-randomized trial. Arch. Intern. Med. 2009; 169(8):771-780. DOI: 10.1001/archinternmed.2009.51.

10. Shah NR, Seger AC, Seger DL, Fiskio JM, Kuperman GJ, Blumenfeld B, Recklet EG, Bates DW, Gandhi TK. Improving acceptance of computerized prescribing alerts in ambulatory care. J. Am. Med. Inform. Assoc. 2006; 13(1):5-11. DOI: 10.1197/jamia.M1868

11. Mekhjian HS, Kumar RR, Kuehn L, Bentley TD, Teater P, Thomas A, Payne B, Ahmad A. Immediate benefits realized following implementation of physician order entry at an academic medical center. J. Am. Med. Inform. Assoc. 2002; 9(5):529-539. DOI: 10.1197/jamia.M1038.

12. Poon EG, Blumenfeld B, Hamann C, Turchin A, Graydon-Baker E, McCarthy PC, Poikonen J, Mar P, Schnipper JL, Hallisey RK, Smith S, McCormack C, Paterno M, Coley CM, Karson A, Chueh HC, Van Putten C, Millar SG, Clapp M, Bhan I, Meyer GS, Gandhi TK, Broverman CA. Design and implementation of an application and associated services to support interdisciplinary medication reconciliation efforts at an integrated healthcare delivery network. J. Am. Med. Inform. Assoc. 2006; 13(6):581-592. DOI: 10.1197/jamia.M2124.

13. Patterson ME, Marken P, Zhong Y, Ketcherside W. Comprehensive electronicmedical record implementation levels not associated with 30-day all-cause readmissions within Medicare beneficiaries with heart failure. Appl. Clin. Inform. 2014; 5(3):670-684. DOI: 10.4338/ACI2014-01-RA-0008.

14. Canêo PK, Rondina JM. Prontuário Eletrônico do Paciente: conhecendo as experiências de sua implantação. J. Health Inform. 2014; 6(2):67-71. 
15. Farias JS, Guimarães TA, Vargas ER, Albuquerque PHM. Adoção de prontuário eletrônico do paciente em hospitais universitários de Brasil e Espanha. A percepção de profissionais de saúde. RAP. 2011; 45(5):1303-1326. DOI: $10.1590 / \mathrm{S} 0034-76122011000500004$.

16. Galanter WL, Didomenico RJ, Polikaitis A. A trial of automated decision support alerts for contraindicated medications using computerized physician order entry. J. Am. Med. Inform. Assoc. 2005; 12(3):269-274. DOI: 10.1197/jamia.M1727.

17. Marin HF. Sistemas de informação em saúde: considerações gerais. J. Health Inform. 2010; 2(1):20-24.

18. Correr CJ, Otuki MF, Soler O. Assistência farmacêutica integrada ao processo de cuidado em saúde: gestão clínica do medicamento. Rev. Pan-Amaz. Saude. 2011; 2(3):41-49. DOI: 10.5123/S2176-62232011000300006.

19. Kuperman G, Gibson RF. Computer physician order entry: benefits, costs, and issues. Ann. Intern. Med. 2003; 139(1):31-39. DOI: 10.7326 / 0003-4819-139-1200307010-00010
20. Allard J, Carthey J, Cope J, Pitt M, Woodward S. Medication errors: causes, prevention and reduction. Br. J. Haematol. 2002; 116(2):255-265. DOI: 10.1046/j. 1365-2141.2002.03272.x

21. Nuckols TK, Smith-Spangler C, Morton SC, Asch SM, Patel VM, Anderson LJ. The effectiveness of computerized order entry at reducing preventable adverse drug events and medication errors in hospital settings: a systematic review and meta-analysis. Syst. Rev. 2014; 3:56. DOI: $10.1186 / 2046-4053-3-56$

22. Devine EB, Hansen RN, Wilson-Norton JL, Lawless NM, Fisk AW, Blough DK, et al. The impact of computerized provider order entry on medication errors in a multispecialty group practice. J. Am. Med. Inform. Assoc. 2010; 17:78-84. DOI: 10.1197 / jamia.M3285

23. Cassiani, SHDB. Erros na medicação: estratégias de prevenção. Rev. Bras. Enferm. 2000; 53(3):424-430. DOI: $10.1590 / \mathrm{S} 0034-71672000000300010$ 